\title{
More Efficient Photolithographic Synthesis of DNA-Chips by Photosensitization
}

\author{
D. Wöll, ${ }^{1}$ S. Walbert, ${ }^{1}$ K.-P. Stengele, ${ }^{2}$ R. Green, ${ }^{3}$ T. Albert, ${ }^{3}$ \\ W. Pfleiderer, ${ }^{1}$ and U. E. Steiner ${ }^{1, *}$ \\ ${ }^{1}$ University of Konstanz, Konstanz, Germany \\ ${ }^{2}$ Chemogenix GmbH, Pleiskirchen, Germany \\ ${ }^{3}$ Nimblegen Systems Inc., Madison, Wisconsin, USA
}

Light-induced release of photolabile protecting groups is a key step in the photolithographic in-situ synthesis of oligonucleotides in the production of high-density DNA-chips. ${ }^{[1,2]}$ Protecting groups of the o-nitrophenyl type that are preferentially used for this purpose have rather low absorption coefficients at $366 \mathrm{~nm}$, the wavelength of the most conveniently used mercury line. The weak absorptivity of these compounds leads to principal limitations of their light sensitivity. Since they have photoreactive triplet states, however, this problem can be overcome by introducing a triplet sensitizer that efficiently absorbs the light and transfers the electronic energy to the reactive protecting group.

The sensitized photodeprotection was studied in detail for the o-nitrophenyl2-propoxycarbonyl (NPPOC) group ${ }^{[3]}$ where the photo cleavage occurs via $\beta$-elimination of the corresponding nitrostyrene derivative as shown in the scheme. The first step of this reaction is photochemically initiated intramolecular $\mathrm{H}$-atom transfer from the $\alpha$-position to the nitro group whereby an aci-nitro compound is formed ${ }^{[3]}$. Protolytic dissociation of the aci-nitro compound is followed by the $\beta$-elimination from the aci-nitro anion. As an example of the time-resolved

*Correspondence: U. E. Steiner, Universität Konstanz, Fachbereich Chemie, Universitätsstrasse 10, 78457 Konstanz, Germany; Fax: +49 753188 3138; E-mail: ulrich.steiner@unikonstanz.de. 


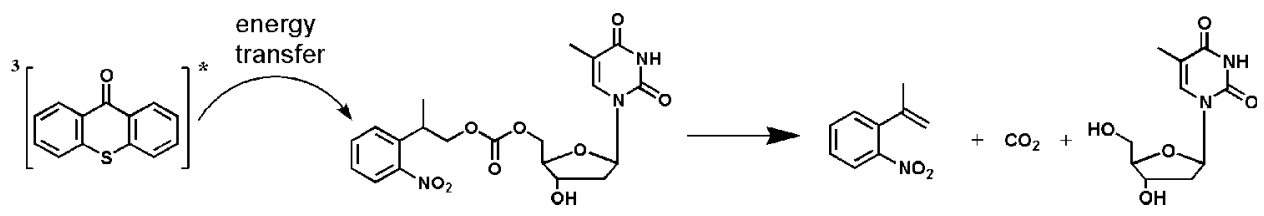

observation of triplet sensitized formation of the intermediate aci-nitro compound, laser flash spectroscopic signals at $420 \mathrm{~nm}$, the absorption maximum of the aci-nitro form, are shown in Fig. 1. Here the rise time of the aci-nitro form related transient absorption is seen to decrease with increasing concentration of the photolabile NPPOC-protected thymidine (NPPOC-T) functioning as the triplet energy acceptor in this case.

Various potential sensitizers such as acridone and thioxanthone were investigated by using absorption, fluorescence and laser flash spectroscopy. Furthermore, continuous irradiations followed by HPLC separation and characterization of the peaks by absorption spectroscopy were used to determine the kinetics of reactants and products and to assess the quantum yields of the photosensitized reactions. Thioxanthone turned out to be the most suitable sensitizer for the o-nitrophenyl-2-propoxycarbonyl (NPPOC) group. In homogenous solutions (cf. Fig. 2) the light sensitivity could be enhanced by as much as a factor of 10, while in terms of overall deprotection yield and avoidance of side reactions the photosensitized reaction was as efficient as the direct photoreaction.

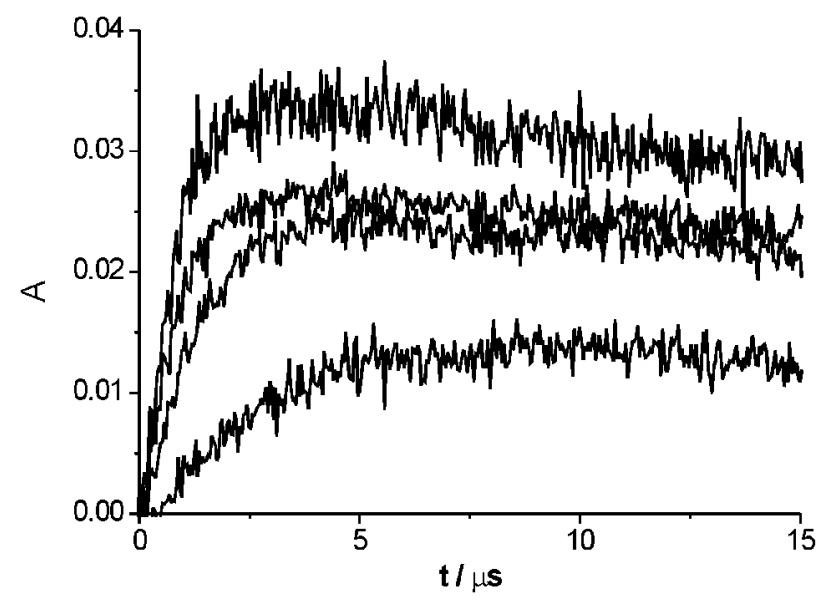

Figure 1. Transient absorption signals observed at $420 \mathrm{~nm}$ upon laser flash photolysis at $355 \mathrm{~nm}$ of an acetonitrile solution of 2-ethylthioxanthone containing increasing concentrations of NPPOC-T (curves from below: 0.135, 0.247, 0.358, 0.480 M). The transient absorption signals are corrected for the overlapping triplet-triplet absorption of 2-ethylthioxanthone and absorbance contributions resulting from direct photolysis of NPPOC-T. 


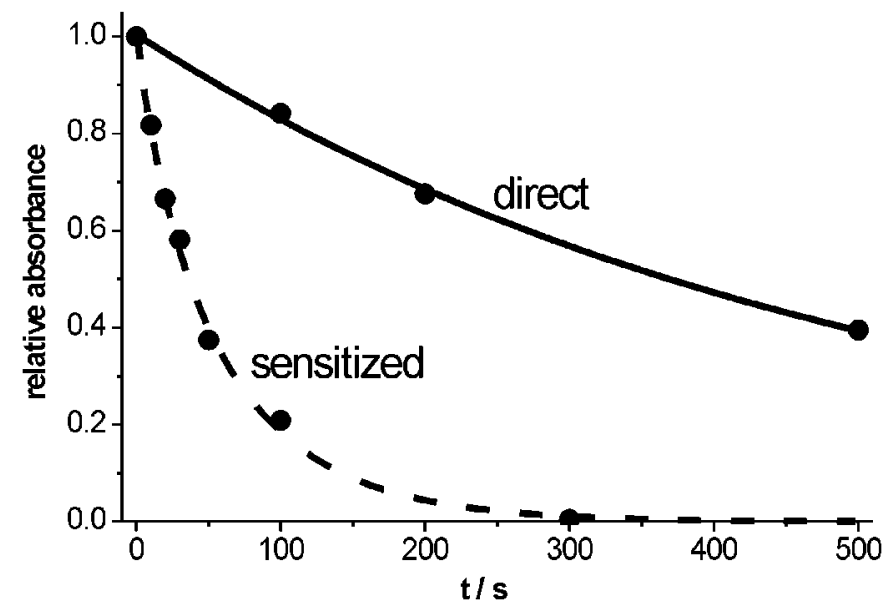

Figure 2. Kinetics of direct and thioxanthone sensitized photocleavage of NPPOC-T in acetonitrile. The concentrations of NPPOC-T and thioxanthone were $0.091 \mathrm{mM}$ and $0.113 \mathrm{mM}$, respectively. The reaction progress was followed by HPLC analysis and monitoring the UV absorbance of the peak of uncleaved NPPOC-T.

Photosensitization has also been tested for high-density DNA-chip synthesis and has been found to significantly reduce the cycle time of the synthesis without significant reduction in the quality of the chip. ${ }^{[4]}$ Characteristic kinetic traces are shown in Fig. 3. It is particularly worth of note that the

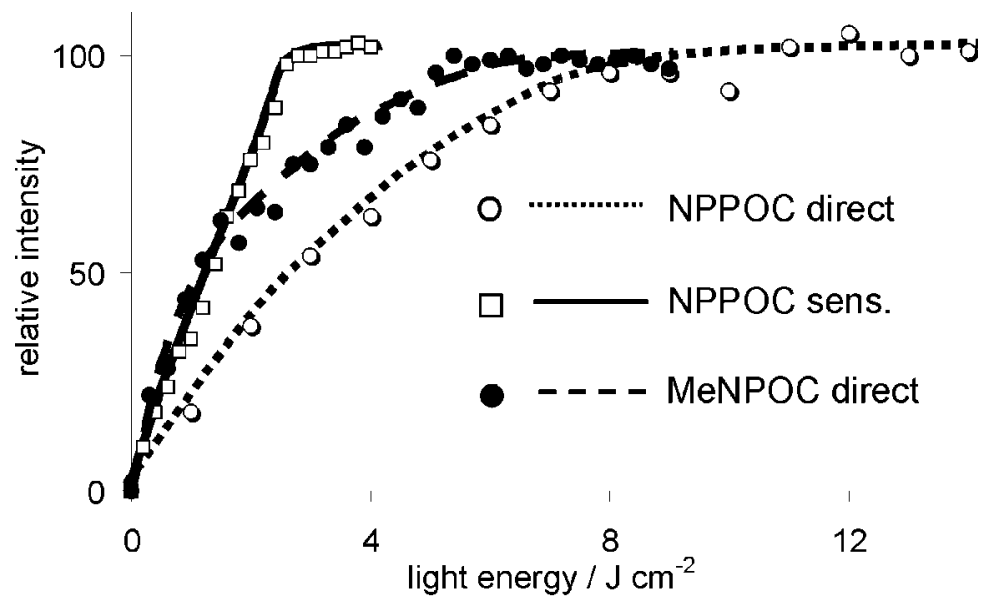

Figure 3. Kinetics of direct and sensitized photodeprotection of thymidine on a DNA chip. A single layer of protected thymidin (NPPOC-T or MeNPOC-T) was bound to a silanized microscope slide. After illumination with the given dose, the fluorescence label Cy3 amidite was coupled to the array. The observed fluorescence intensity provides a relative measure of free hydroxyl groups. 
reaction order of the sensitized reaction on the chips is close to zero, which is particularly favorable because it shortens the necessary illumination time.

\section{REFERENCES}

1. Fodor, S.P.A.; Read, J.L.; Pirrung, M.C.; Stryer, L.; Lu, A.T.; Solas, D. Lightdirected, spatially adressable parallel chemical synthesis. Science 1991, 251 (4995), 767-773.

2. Singh-Gasson, S.; Green, R.D.; Yue, Y.; Nelson, C.; Blattner, F.; Sussmann, M.R.; Cerrina, F. Maskless fabrication of light-directed oligonucleotide microarrays using a digital micromirror array. Nature Biotechn. 1999, 17 (10), 974-978.

3. Giegrich, H.; Eisele-Bühler, S.; Hermann, C.; Kvasyuk, E.; Charubala, R.; Pfleiderer, W. New photolabile protecting groups in nucleoside and nucleotide chemistry - Synthesis, cleavage mechanisms and applications. Nucleosides \& Nucleotides 1998, 17 (9-11), 1987-1996.

4. Steiner, U.E.; Walbert, S.; Wöll, D. Verfahren zur Abspaltung von labilen funktionellen Gruppen aus chemischen Verbindungen. German patent DE 10209 203. 6-45. 